\title{
Stress Resistance in the Naked Mole-Rat: The Bare Essentials - A Mini-Review
}

\author{
Kaitlyn N. Lewis ${ }^{a, b}$ James Mele ${ }^{a, c}$ Peter J. Hornsby ${ }^{a, c}$ Rochelle Buffenstein ${ }^{a, c}$ \\ a Barshop Institute for Longevity and Aging Studies, and Departments of ${ }^{b}$ Cellular and Structural Biology and \\ 'Physiology, University of Texas Health Science Center at San Antonio, San Antonio, Tex., USA
}

\section{Key Words}

Naked mole-rat $\cdot$ Longevity $\cdot$ Stress resistance $\cdot$ Nrf2 $\cdot$ p53

\begin{abstract}
Background: Studies comparing similar-sized species with disparate longevity may elucidate novel mechanisms that abrogate aging and prolong good health. We focus on the longest living rodent, the naked mole-rat. This mouse-sized mammal lives $\sim 8$ times longer than do mice and, despite high levels of oxidative damage evident at a young age, it is not only very resistant to spontaneous neoplasia but also shows minimal decline in age-associated physiological traits. Objectives: We assess the current status of stress resistance and longevity, focusing in particular on the molecular and cellular responses to cytotoxins and other stressors between the short-lived laboratory mouse and the naked mole-rat. Results: Like other experimental animal models of lifespan extension, naked mole-rat fibroblasts are extremely tolerant of a broad spectrum of cytotoxins including heat, heavy metals, DNA-damaging agents and xenobiotics, showing $\mathrm{LD}_{50}$ values between 2- and 20 -fold greater than those of fibroblasts of shorter-lived mice. Our new data reveal that naked mole-rat fibroblasts stop proliferating even at low doses of toxin whereas those mouse fibroblasts that survive treatment rapidly re-enter the cell cycle and may proliferate with DNA damage. Naked mole-rat fibroblasts also show significantly higher constitutive levels of both $\mathrm{p} 53$
\end{abstract}

(C) 2012 S. Karger AG, Basel

0304-324X/12/0585-0453\$38.00/0

Fax +4161306 1234

E-Mail karger@karger.ch

www.karger.com
Accessible online at:

www.karger.com/ger and Nrf2 protein levels and activity, and this increases even further in response to toxins. Conclusion: Enhanced cell signaling via $\mathrm{p} 53$ and Nrf2 protects cells against proliferating with damage, augments clearance of damaged proteins and organelles and facilitates the maintenance of both genomic and protein integrity. These pathways collectively regulate a myriad of mechanisms which may contribute to the attenuated aging profile and sustained healthspan of the naked mole-rat. Understanding how these are regulated may be also integral to sustaining positive human healthspan well into old age and may elucidate novel therapeutics for delaying the onset and progression of physiological declines that characterize the aging process.

Copyright $\odot 2012$ S. Karger AG, Basel

\section{Introduction}

Comparative biology of aging assesses divergent responses in species with disparate longevity and is a useful tool in identifying novel and conserved mechanisms that may contribute to healthspan and longevity, mechanisms that may not be evident using traditional experimentally manipulated animal models. In non-volant, non-aquatic mammals, species longevity is dependent on the size of the organism such that a doubling of species body mass results in a $16 \%$ increase in longevity [1]. There are, however, several species that do not conform to this relation- 
ship and comparative biology of aging exploits those deviant species. While death is not always due to intrinsic mortality, maximum lifespan potential nevertheless is a useful indicator of the rate of aging, and species that live longer than predicted may reveal evolved mechanisms that delay either the onset or rate of aging. Long-lived species are defined as those organisms that live more than twice as long as predicted by body mass (i.e. have a longevity quotient $>2$ ) and are particularly useful in highlighting mechanisms that may be involved in retarding aging processes. Both humans and naked mole-rats (Heterocephalus glaber) are extremely long-lived for their body size, with longevity quotients ranging between 4 and 10 depending upon the allometric equation used [2]. Although few studies have utilized a comparative approach in longevity research [3-5], a greater number of comparative studies are revealing common mechanisms among long-lived species, which may contribute to sustained good healthspan and long lifespan.

\section{Naked Mole-Rats and Aging Research}

The longest-lived rodent known, the naked mole-rat, has rapidly emerged as a natural model of extreme longevity based upon its more than 30-year maximum lifespan and prolonged maintenance of positive healthspan [6]. This $\sim 35$ g rodent lives 8 times longer than similar sized mice and maintains good health for at least $66 \%$ of its life, which would be equivalent to 80 -year-old humans exhibiting a 30-year-old 'biological age' [6].

During their long lifespan, our laboratory has reported that naked mole-rats show a minimal decline in most aspects of healthspan for at least the first 20 years. They show unchanged body composition and bone density with age in addition to minimal changes in physical activity and no obvious signs of cognitive decline. Similarly, basal metabolic rate and biochemical activity of mitochondrial and antioxidant enzyme activity are unchanged [6].

This species is also able to maintain a healthy reproductive status up until death, with our oldest reproductive female dying at 27 years of age. Sustained reproductive capacity and high fertility throughout life provides further evidence of sustained healthspan as does the finding that naked mole-rats in captivity show no increase in mortality rate from 2 to 20 years of age. The lack of an actuarial increase in mortality may reflect the marked resistance of naked mole-rats to the most common cause of mortality reported in laboratory rodents, cancer. In contrast to what has been observed in naked mole-rats, $70 \%$ of one particular laboratory mouse strain (C57BL/6) mice die of cancer and many more show signs of lesions and small non-lethal tumors [7]. Further evidence of pronounced cancer resistance was established by attempts at oncogenic transformation of naked mole-rat cells. Unlike cells of all other mammals studied to date, naked mole-rat skin fibroblasts display marked resistance to transformation with Ras ${ }^{\mathrm{G} 12 \mathrm{~V}}$ and SV40 large $\mathrm{T}$ antigen [8]. Naked mole-rat cells also readily undergo growth arrest under suboptimal culture conditions whereas, when maintained optimally, cells grow to high densities. As such, suboptimal conditions may result in apparent contact inhibition and this cellular response may also be integral to their cancer resistance [9]. Both genetic and proteomic integrity appear to be maintained with age, between the ages of 2 and 26 years. Naked molerats show minimal or no differences in a wide array of biochemical processes and protein homeostasis; this is in sharp contrast to the age-related differences commonly seen in laboratory-raised mice [6]. This sustained genomic maintenance may contribute substantially to the extreme longevity of naked mole-rats.

Even at a young age, naked mole-rats have surprisingly high levels of oxidative damage compared to mice. However, oxidative damage to either lipids or proteins does not accrue with age in the mole-rats as they do in aged mice [3]. Clearly, naked mole-rats have mechanisms in place to protect against stressors and prevent further oxidative damage accrual and the translation of these high levels of damage into a decline in normal function [3].

Naked mole-rats show many common features with experimental models of long-lived mice (i.e. dwarf and calorically restricted (CR) mice). Like dwarf mice, naked mole-rats show decreased body temperature, fasting glucose and metabolic activity as well as enhanced tolerance of exposure to oxidative stressors compared to similarly sized wild-type CB6F1 hybrid mice $[10,11]$. Multiple mechanisms have been proposed that may contribute to the traits evident in models of extended longevity and many of these may be considered highly adaptive to life underground.

Naked mole-rats are eusocial mammals, that, like bees and other social insects, live in colonies of up to 300 animals, with a strict division of labor culminating in the presence of a single breeding female who suppresses the sexual maturity of the subordinates in the colony. Subordinates within the colony maintain the burrow system and care for young in addition to foraging for food. These 
small, pink hystricognath rodents, more closely related to guinea pigs, porcupines and rock rats than to traditional laboratory mice and rats, are naturally found in the eastern horn of sub-Saharan Africa. Here, they live communally in an underground maze of tunnels and chambers up to $8 \mathrm{ft}$ beneath the soil surface [12]. Living underground protects the mole-rats from extreme changes in weather and temperature in addition to protection from predation. However, it poses other challenges associated with life in a dark dank environment with poor gas and heat exchange [13]. Not surprisingly, they therefore have low basal metabolic rates and resting body temperatures [14].

Covered in only sparse, tactile hairs rather than an insulatory coat of fur, naked mole-rats are thermally labile when housed on their own outside their warm humid equatorial milieu. Naturally living in large groups they are also extremely tolerant of hypoxia and hypercapnia [13]. Naked mole-rats acquire all their nutrients and water by feeding off roots, bulbs, corms and tubers found underground [15]. Often these underground plant storage components have allelochemicals or secondary defenses (e.g. cyanogenic glucosides, alkaloids and phenols) to protect themselves against exploitation as food sources by herbivores. As such, naked mole-rats, like many other herbivores, may have co-evolved mechanisms to counteract these toxins, in addition to their tolerance of hypercapnia and thermolability. These are also integral to their extraordinary longevity.

Multiple mechanisms have been hypothesized for the large disparity in longevity relative to body size and overall health of naked mole-rats. We will evaluate the contribution of cytoprotective mechanisms and stress resistance to aging, healthspan and longevity, of a wide variety of species with disparate longevity, focusing particularly on the naked mole-rat.

\section{Stress Resistance and Aging}

Aging is a multicausal process with conserved characteristics across a variety of species that leads to increasing susceptibility to both endogenous and external challenges. These include a decline in genomic integrity, an accumulation of oxidative damage, increases in various pathologies and, most importantly, an increase in mortality [16]. Perturbation of multiple physiological mechanisms contributes to the key characteristics that defile the aging profile. These include less efficient DNA repair mechanisms, increased free radical production and oxidative stress, as well as a decline in proteomic function and in-

Stress Resistance in the Naked Mole-Rat: The Bare Essentials tegrity. Oxidative stress is widely regarded as causally linked to the aging process and is also implicated in many age-associated diseases such as neurodegeneration, cancer and cardiovascular disease, and ultimately, death.

A great deal of published data supports the oxidative stress theory of aging [17], although recent research focusing in particular on genetically manipulated mice that either over- or underexpress key antioxidants has raised doubts over the importance of reactive oxygen species and their metabolism as a determinant of longevity [18]. Comparative data across several vertebrate phyla also yield equivocal support for this theory. Data from birds and reptiles, as well as long-lived rodent models, show higher levels of free radical production or oxidative damage accrual, when compared to shorter-lived species. This does not appear to impact their maximum species lifespan or their healthspan [3].

Species differences in resistance to oxidative and other endogenous and/or environmental stressors correlate with maximum species lifespan potential. Enhanced stress resistance has been shown in skin fibroblasts from multiple long-lived species after a variety of toxic insults, including heavy metals, DNA-damaging agents, heat and dietary interventions $[4,5,11]$. This type of cytoprotection moves beyond neutralization of oxidative stress but also encompasses other exogenous chemical stressors and supports the premise that the degree of endogenous oxidative stress is not a determinant of species longevity. Rather, longevity is influenced by how the species responds to mitigate the impact of that particular stressor.

Considerable evidence has now accumulated showing that cytoprotection and stress resistance may be a significant contributing factor to a positive healthspan and extended lifespan. Indeed experimental studies of extended longevity, based upon both genetic manipulations [19] and dietary restriction [20], together with comparative studies among species with disparate longevity $[4,5$, 11] have all revealed that resistance to stressors is a common trait of longer-lived organisms.

Stress resistance is rapidly emerging as a prominent predictor of both good healthspan and longevity. Intuitively, an increase in stress resistance at a cellular and organismal level would not only result in augmented resistance to environmental toxins, but also enhanced protection against inflammation, neurodegeneration and cancer, diseases that are often associated with aging. Increased toxin resistance may also contribute to a more stable proteome and genome, thereby retarding senescence and aging. Not surprisingly, data published in both invertebrates and vertebrates show that increased stress 
Table 1. Fibroblasts from the longest-lived rodent, the naked mole-rat, are significantly more resistant to a multitude of stressors in vitro including heavy metals and chemotherapeutic agents, compared to those from the similarly sized but shorter-lived C57Bl/6 mouse

\begin{tabular}{lcll}
\hline Compound & $\begin{array}{l}\text { Mouse } \\
\left(\mathrm{LD}_{50}\right)\end{array}$ & $\begin{array}{l}\text { Naked mole- } \\
\text { rat }\left(\mathrm{LD}_{50}\right)\end{array}$ & $\begin{array}{l}\text { Fold increase (from } \\
\text { mouse to naked mole-rat) }\end{array}$ \\
\hline Cadmium & 12.5 & 50 & 4 \\
Zinc & 12.5 & 30 & 2.4 \\
Chromium & 5 & 25 & 5 \\
Adriamycin & 1.25 & 20 & 16 \\
Camptothecin & 2 & 30 & 15 \\
\hline
\end{tabular}

$\mathrm{LD}_{50}$ values presented in this table are from fibroblasts maintained in MEM to which $10 \%$ fetal bovine serum was added and incubated at $37^{\circ} \mathrm{C}$. Cells were exposed to the heavy metal (cadmium, chromium and zinc) for $2 \mathrm{~h}$ and to the chemotherapeutic (camptothecin, adriamycin) agent for $6 \mathrm{~h}$. Viability was measured $24 \mathrm{~h}$ later by MTT reduction and quantification at $550 \mathrm{~nm}$. Absorbance from untreated controls was set to 1.0; absorbance from treated groups was then calculated as (relative) viability. These data show that NMR cells are more resistant to this diverse set of cytotoxins for the $\mathrm{LD}_{50}$ values for naked mole-rat cells range between 2.4- and 16-fold higher than those of mouse cells.

resistance has a positive relationship with long lifespan $[4,11,21-24]$. The presence of regulated biochemical mediated protection is also evident in that in vitro cytoprotection can be attained by incubating multiple cell types with serum from CR rhesus macaques [25] or with serum from CR humans [20]. In the latter study, cells from mice showed a $25 \%$ increase in heat resistance and this was attributed to molecular chaperones present in the serum.

A correlation among in vitro stress resistance and species longevity is also observed in fibroblasts from all phylogenetic groups studied to date, including birds [24], bats [26], domesticated mammals and a diverse array of rodents $[4,5,11]$. Stress resistance is broad based and not restricted to oxidative stressors including diquat and hydrogen peroxide, but extends to heavy metals and chemotherapeutic agents that directly damage DNA as well. Resistance to stressors in long-lived species has been used as an exemplar of the somatic theory of aging positing that longer-lived species have invested considerable resources in mechanisms to better protect and maintain their somatic tissues than do shorter-lived species $[4,27]$. Pro-mislow [28], in re-evaluating published data, observed that the correlation in cellular tolerance of toxins with species longevity was primarily due to body size differences. As many of these studies are performed using fibroblasts in vitro, observed interspecific differences may also be due to divergent optimal culture conditions for the various species. A previous study assessed stress resistance of naked molerat cells when both mouse and naked mole-rat fibroblasts were maintained at $33^{\circ} \mathrm{C}$, approximating the preferred body temperature of a naked mole-rat, and under serumstarved conditions. This study under suboptimal conditions for the heterogeneous four-way cross mouse cell line revealed that naked mole-rat cells are more resistant to different types of stressors including cadmium, heat, oxidative stress (e.g. diquat), the DNA-damaging agent methyl methanesulfonate (MMS) and starvation [11]. We have extended this study to include assessments under a variety of conditions (e.g. in complete media), including those more favorable for mouse cells and in response to additional toxins, and found that naked mole-rats consistently maintain better protection against a broad array of toxins (table 1), independent of culture conditions.

\section{Naked Mole-Rats and Stress Resistance}

Current data from our laboratory show that the $\mathrm{LD}_{50}$ from naked mole-rat fibroblasts is 2 - to 20 -fold higher than fibroblasts from mice after 2-6-hour treatments with heavy metals and DNA-damaging agents (table 1). Not only are these $\mathrm{LD}_{50}$ values higher, but, under conditions considered more optimal for mouse cells than naked mole-rat cells, naked mole-rat fibroblasts still show superior resistance to toxins.

\section{Culture Conditions and Toxicity Studies}

Toxicity studies have demonstrated that culture conditions, especially prior to toxin treatment, can influence in vitro cellular resistance [29]. Minimum essential medium has been shown to better protect growth-sensitive cell types against oxidative stress [30]. We have specifically conducted our studies in minimum essential medium, which would eliminate potentially deleterious effects resulting from supraphysiologically nutrient-rich media.

We have found that skin fibroblasts of naked mole-rats grow significantly better at $32^{\circ} \mathrm{C}$ than at $37^{\circ} \mathrm{C}$. This is most likely attributed to the low resting body temperature of $\sim 32^{\circ} \mathrm{C}$ observed in both field-caught and laboratory-maintained animals [14]. We have compared both inter- and intraspecific differences in cell viability in response to heavy metal toxicity at both 32 and $37^{\circ} \mathrm{C}$. Increasing the temperature increases the sensitivity to metal toxicity, such that the mouse fibroblast $\mathrm{LD}_{50}$ with cadmium declines 2.79 -fold when maintained at a $5^{\circ} \mathrm{C}$ 


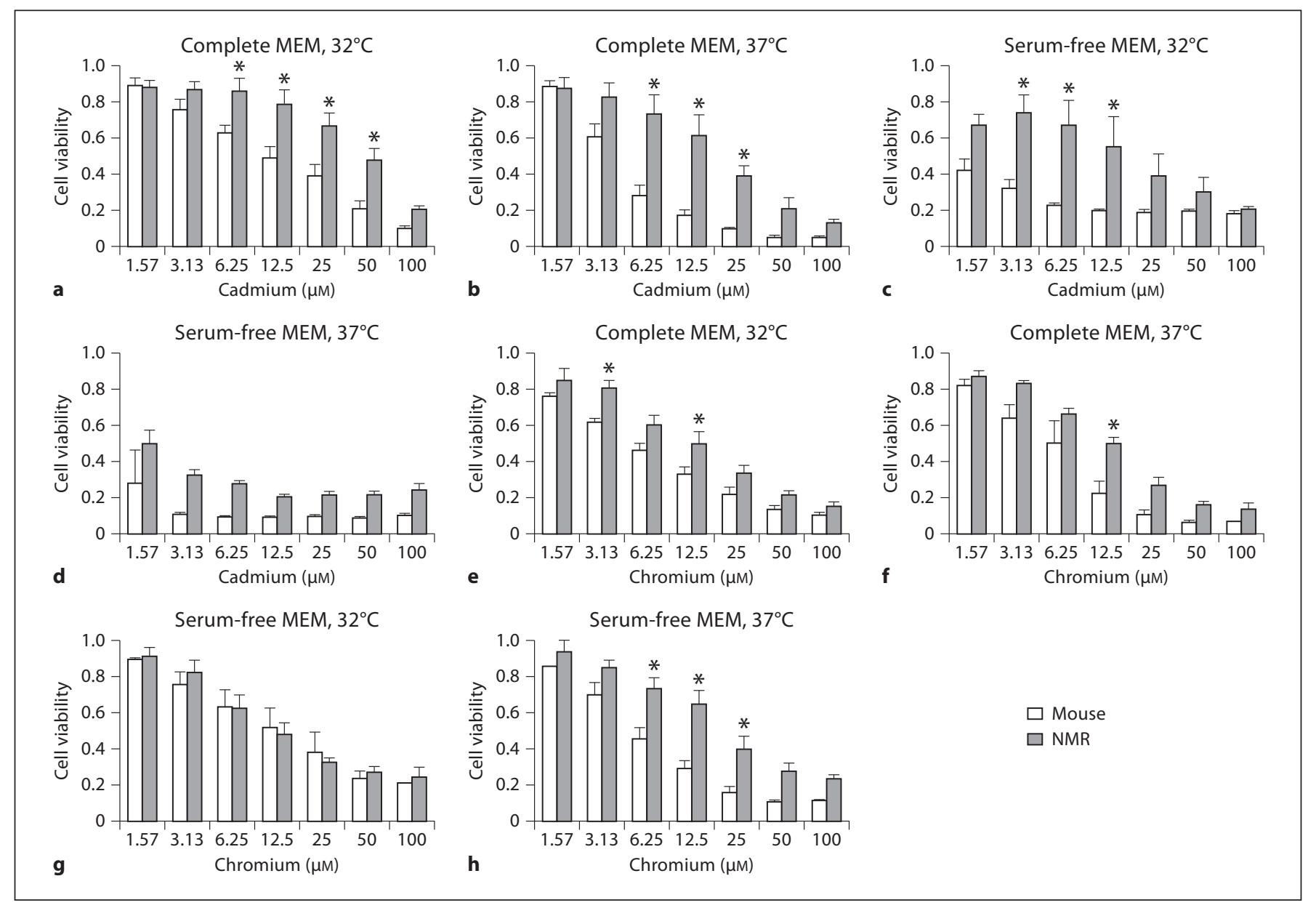

Fig. 1. In vitro culture conditions can play a role in the cytotoxicity of cadmium (a-d) and chromium (e-h) in naked mole-rat and mouse fibroblasts. Under serum-fed conditions, alterations in temperature do not have a large effect on naked mole-rat fibroblast survival $24 \mathrm{~h}$ after treatment with various doses of cadmium and chromium for $2 \mathrm{~h}(\mathbf{a}, \mathbf{b}, \mathbf{e}, \mathbf{f})$. When cells are treated with identical doses of heavy metals under serum-free conditions for $2 \mathrm{~h}$, temperature impacts the resistance of naked mole-rat cells to cadmium; however it may slightly enhance resistance to chromium at $37^{\circ} \mathrm{C}$. Most importantly, naked mole-rat fibroblasts demonstrate greater resistance to both heavy metals compared to mouse fibroblasts under all but one culture condition (serum-free medium, $32^{\circ} \mathrm{C}$ ) tested. higher temperature and treated in complete medium. In contrast, the naked mole-rat $\mathrm{LD}_{50}$ values are unaffected by the elevation in incubation temperature and, in keeping with their thermal tolerance of a wide variety of ambient conditions, naked mole-rat fibroblasts generally maintain superior resistance to metal toxicity compared to those of mice. Interspecies differences in response to chromium and cadmium exposure (fig. 1) are attenuated when cultivated at the lower temperature. Particularly, mouse fibroblasts show greater resistance to heavy metal toxicity when maintained at the non-physiological, lower ambient temperature compared to mouse fibroblasts cultured at $37^{\circ} \mathrm{C}$. Like all biochemical reactions, tempera- ture influences the uptake and metabolic processing of toxins and it is likely that mouse cells, in keeping with their lower metabolic rate induced by the lower temperature, have reduced metal uptake at $32^{\circ} \mathrm{C}$ and that this contributes to the observed improvement in cell viability. Comparative studies that fail to properly control for optimal cultivation may thus yield spurious findings.

Cultivation of fibroblasts in low serum media prior to metal exposure dramatically reduces the cell viability in a temperature-specific fashion (fig. 1). Serum contains a multitude of proteins important for stimulating growth and attachment of cells and has been shown to modulate the rate of free radical production in porcine lung fibro- 
blasts and aortic endothelial cells, affecting their overall maximum population doubling attainable in vitro [31]. Previous reports show that media containing serum provided greater cytoprotection than low serum media against metal toxicity and that serum deprivation is actually considered a source of cellular stress [32]. In only one case, the assessment of chromium toxicity, serum deprivation on mouse fibroblasts had a beneficial effect on survival against metal toxicity (increasing the $\mathrm{LD}_{50} 3.25$ fold, from 5.569 to $18.11 \mu \mathrm{M}$; fig. 1). These findings suggest that serum deprivation is not critical for evaluating comparative stress resistance among species.

Overall, our results in low serum media are consistent with those previously published using cadmium [11], in that the naked mole-rat fibroblast $\mathrm{LD}_{50}$ is double that observed in mice for cadmium toxicity (fig. 1). Our study reveals that cultivar conditions can greatly influence the $\mathrm{LD}_{50}$ toxin concentration. This last point is particularly evident if one compares the resistance to cadmium toxicity of Snell dwarf mice published in two separate studies $[19,33]$. Both studies found that fibroblasts isolated from long-lived Snell dwarf mutant mice were about 3-fold greater than wild-type littermates, however, interstudy differences in the recorded $\mathrm{LD}_{50}$ values differ by a factor of 5.1. Differences in experimental methodology (i.e. culture conditions) most likely factored into the wide range of values reported and a lack of standardized culture conditions can often hinder interstudy comparisons in a quantitative manner.

Our data reveal that despite the impact of ambient temperature on cell membrane and metabolic properties, as well as the fact that mouse cells were maintained at optimal cultivar conditions whereas naked mole-rat cells at $37^{\circ} \mathrm{C}$ were not, naked mole-rat cells nevertheless were significantly more resilient to cytotoxic stressors than were mouse cells at $37^{\circ} \mathrm{C}$ (fig. 1). These findings suggest a broad-based resilience to both heavy metal toxicity and environmental conditions.

\section{Potential Mechanisms of Stress Resistance}

Clearly, naked mole-rats must have better cellular protective and/or cell cycle regulation mechanisms than do mice in order to facilitate the observed generalized resistance to stressors. Following treatment with high doses of the heavy metal chromium, the majority of surviving fibroblasts from mice enter senescence. In contrast, fibroblasts from naked mole-rats show considerably less staining with $\beta$-galactosidase. If one assumes that this is not due to species differences in responsiveness to $\beta$-galactosidase, but rather a true indication of irreversible senes- cence, this would indicate that fewer naked mole-rat cells irreversibly senesce. This would further support the premise that naked mole-rat cells are more resilient than mouse cells to unfavorable conditions.

We have also evaluated whether or not surviving cells of both species following a cytotoxic insult showed similar proliferative capacity. To assess this we treated the cells with bromodeoxyuridine (BrdU). This synthetic thymidine analog is incorporated into a cell's DNA during the S-phase of the cell cycle when the cell is dividing and is routinely used as a marker of cell proliferation both in vitro and in vivo [34]. Although mouse cells exhibit extensive early cell death, those that do survive rapidly re-enter the cell cycle and continue to proliferate. This finding suggests that mouse cells are likely replicating before complete repair of damage has occurred (fig. 2). In contrast, even at low concentrations of toxins, at which the majority of naked mole-rat cells survive the insult, the proportion of naked mole-rat cells in S-phase (\% BrdU incorporation; fig. 2c) is low. This suggests that those naked mole-rat cells that do survive undergo a prolonged cell cycle arrest in response to genotoxins, and repair damage to their DNA before re-entering the cell cycle, presumably with minimal DNA damage and a reduced chance of mutation induction.

Naked mole-rat fibroblasts exhibit 50 -fold higher levels of p53 even under apparently normal growth conditions (fig. 3a). Nevertheless, p53 activity is highly responsive to genotoxic stressors (fig. 3b). For example, when p53 luciferase reporter activities in mouse and naked mole-rat fibroblasts are compared, naked mole-rat cells show a 15fold increase compared to basal levels whereas only a modest increase (up to 5 -fold) was observed in mouse fibroblasts (fig. 3c, d). This rapid increase in p 53 may reveal enhanced sensitivity in cell-cycle regulation. Constitutive p53 protein levels are at least 50-fold higher in naked mole-rat fibroblasts compared to those in mice. Thus, even though naked mole-rat cells are capable of long-term proliferation in culture, they are poised to undergo growth arrest via $\mathrm{p} 53$ and possibly retinoblastoma protein when they undergo minimal damage. Moreover, we have experimentally shown that if cells in the naked mole-rat fibroblast population do acquire mutations that may be oncogenic, they have yet another protective mechanism; cells enter crisis rather than acquire neoplastic properties (anchorage independence and growth in immunodeficient mice) [8]. We have also found that proteins in tissue homogenates are resistant to unfolding stressors such as heat, urea and iron ascorbate [35], suggesting that enhanced molecular chaperone activity may also be involved 


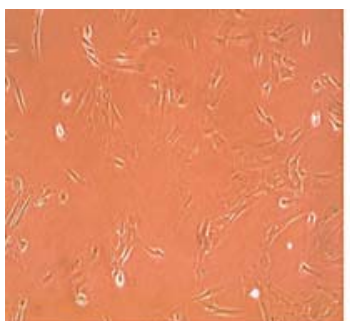

a

Control

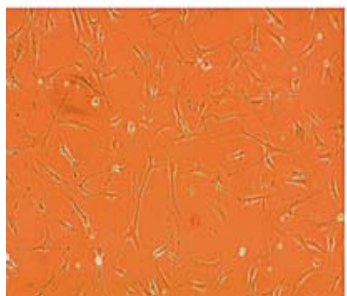

b

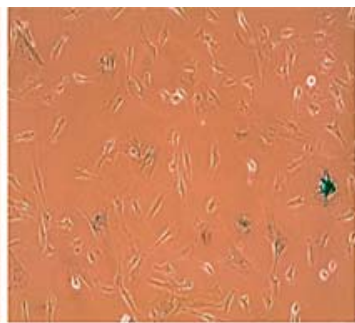

Mouse $\mathrm{LD}_{30}$

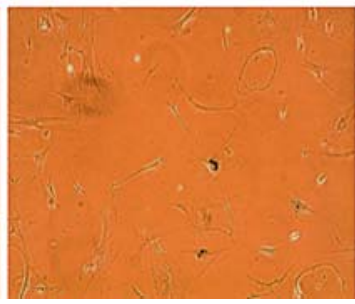

NMR LD 30

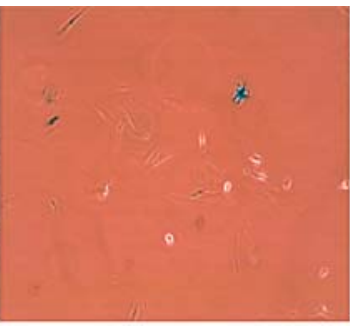

Mouse $\mathrm{LD}_{70}$

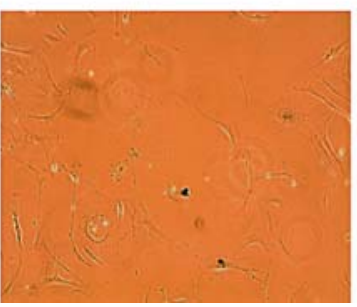

NMR LD 70

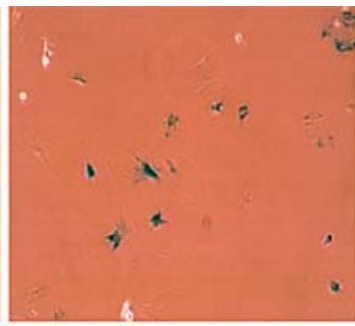

NMR LD 30

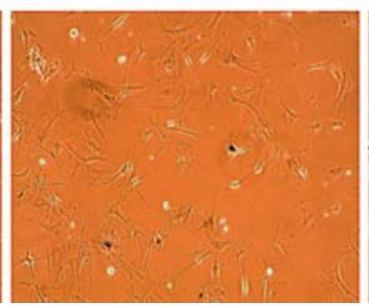

Mouse $\mathrm{LD}_{70}$

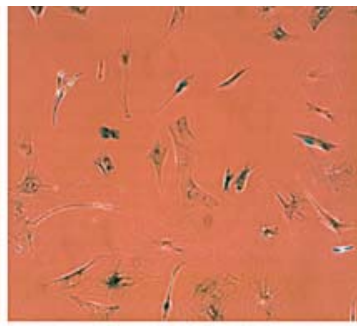

Adriamycin LD $_{50}$

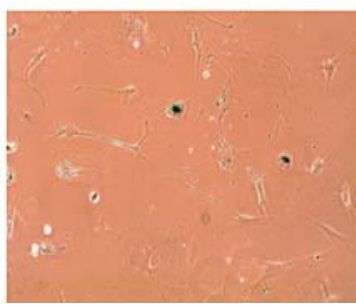

Adriamycin $\mathrm{LD}_{50}$

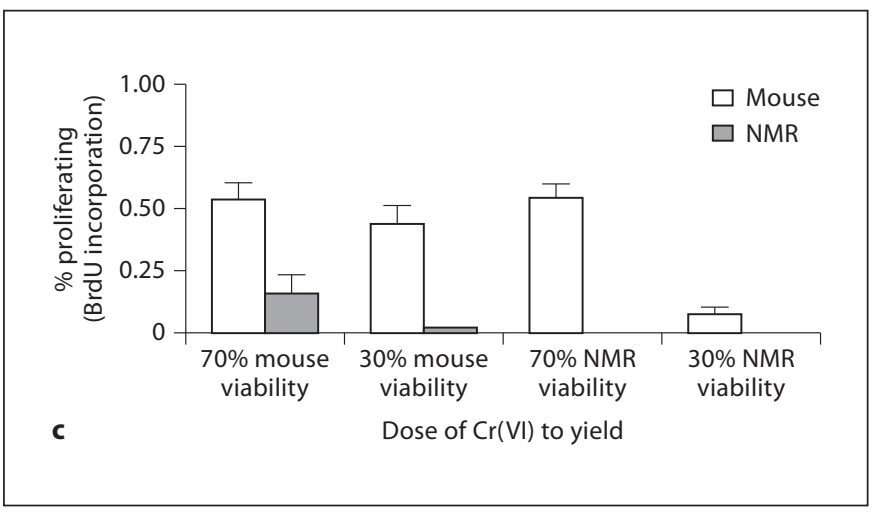

Fig. 2. Naked mole-rat fibroblasts show decreased $\beta$-galactosidase staining after treatment with various doses of chromium and adriamycin compared to mouse fibroblasts $(\mathbf{a}, \mathbf{b})$. This indicates that fewer naked mole-rat cells enter irreversible senescence after toxin treatment and that this species may be utilizing other defense mechanisms to overcome a toxic insult. When the proportion of cells proliferating 5 days after exposure of fibroblasts to doses of chromium equivalent to the $\mathrm{LD}_{70}$ and $\mathrm{LD}_{30}$ for each species is measured by the assessment of BrdU incorporation, naked mole-rats show signs of a prolonged cell cycle arrest whereas those

in the observed profile of superior stress resistance in naked mole-rats. Molecular chaperone activity as well as detoxification processes are regulated by the Nrf2-Keap1signaling cytoprotective pathway [36] and we hypothesize that this pathway is upregulated, largely contributing to the profound stress resistance of the naked mole-rat.

$\mathrm{Nrf2}$ is a transcription factor ubiquitously expressed in all tissues of an organism. Under non-stressed conditions, Nrf2 is bound to Keap1, which with the help of Cullin-3, mouse cells that do survive continue to rapidly proliferate. Naked mole-rat and mouse cells were treated for $2 \mathrm{~h}$ with concentrations of chromium that correspond to these LD values. Cells were then returned to growth media and allowed to recover for 4 days prior to BrdU labeling for $24 \mathrm{~h}$ (c). Naked mole-rat cells lose their proliferative ability even at relatively low chromium concentrations (e.g. mouse $\mathrm{LD}_{70}$ ) whereas mouse cells continue to proliferate in high damage environments (chromium dose corresponding to NMR $L_{70}$ ) that promote genomic instability.

targets Nrf2 for ubiquitination and subsequent degradation via the proteasome. The Nrf2 half-life under basal (non-stressful) conditions is generally short ( $\sim 15 \mathrm{~min}$ ). However, upon a stressful event to the cell, cysteine sensors in Keap1 are modified, altering the conformation of Keap1 and releasing Nrf2. Nrf2 is able to translocate into the nucleus and bind to the antioxidant response element (ARE) to activate the transcription of over 200 cytoprotective genes which include an array of detoxification and 
Fig. 3. Tumor suppressor p53 protein levels, measured by Western blot analyses, are $\sim 50$-fold higher in naked mole-rat fibroblasts compared to those from mice $(\mathbf{a}, \mathbf{b})$. Naked mole-rat fibroblasts expressing the p53 reporter show enhanced sensitivity to high doses of adriamycin, whereas mouse fibroblasts show a more modest increase in p53 activity $24 \mathrm{~h}$ after lower doses of chromium and adriamycin treatment $(\mathbf{c}, \mathbf{d})$.

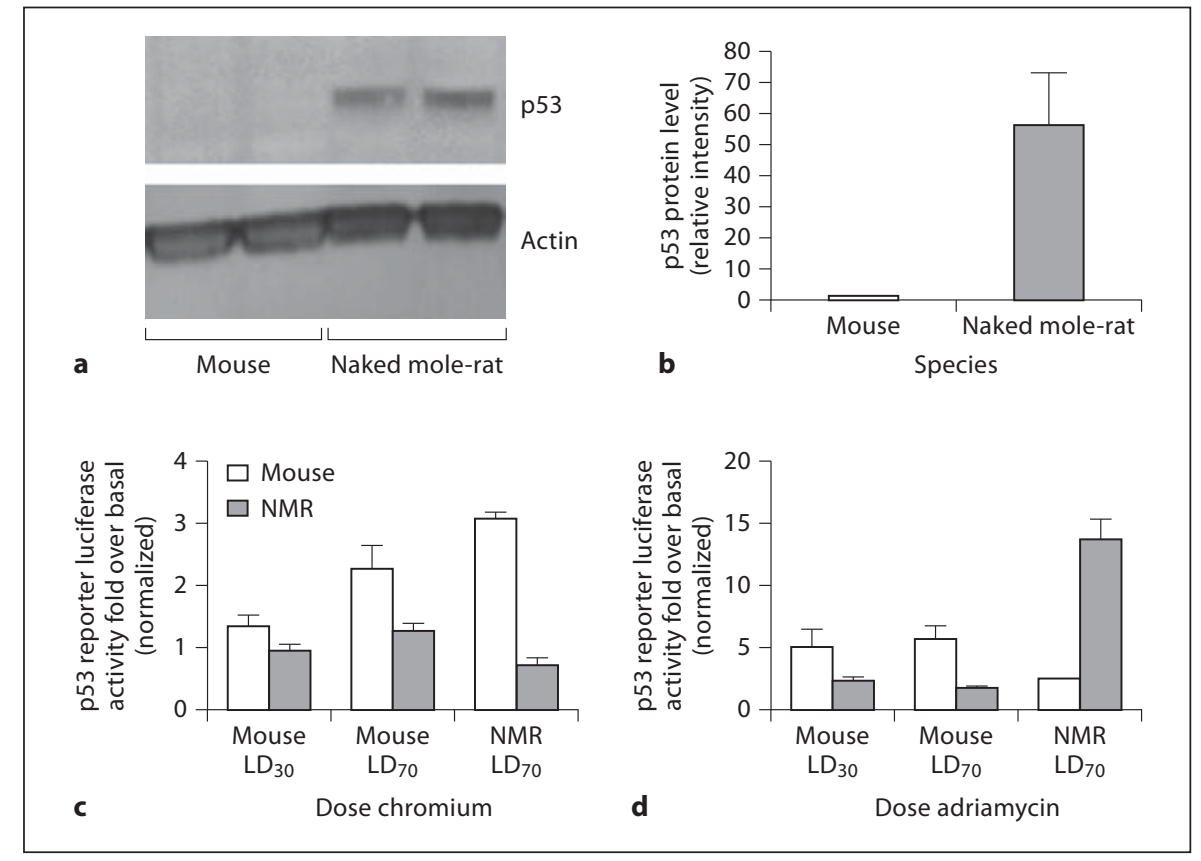

cytoprotective molecules with functions ranging from glutathione and drug metabolism, proteome maintenance and cell cycle regulation. Under stressful conditions, the half-life of Nrf2 is greatly increased ( $\sim 60 \mathrm{~min})$. It is important to note, however, that under basal conditions, some Nrf2 does exist in a free form and is able to constitutively upregulate ARE-activated transcription [37].

Nrf2 has been studied extensively with regard to toxicity [36], cancer [38] and neuroprotection [39] and intuitively should be involved in aging, however research in this area has to a large extent been overlooked. $\mathrm{Nrf} 2$ knockout $\left(\mathrm{Nrf}^{-/-}\right)$mice are more susceptible to induced cancers, neurodegeneration, inflammation, lung and gastrointestinal injury. Fibroblasts and MEFs from $\mathrm{Nrf}^{-/-}$mice are also highly susceptible to many different toxins and stressors in vitro [37].

Evidence has been published in an array of animal models that Nrf2 contributes the stress resistance associated with induced models of longevity. Genetic manipulations of Nrf2 homologues in worms (Skn-1) and flies modulate lifespan and contribute to the lifespan-elongating effects of CR [40]. Similarly, multiple mouse models of longevity show an increase in Nrf2 signaling. CR, methionine-restricted and dwarf mice show an increase in Nrf2-dependent molecules, particularly GSH metabolism [41]. GSH-related enzymes involved in synthesis and drug metabolism, in addition to $\mathrm{Nrf} 2$, have also been shown to decrease in mice and rats as they age [42]. Inter- estingly, a mutation that resulted in a cytotoxic effect resulted in upregulation of the Nrf2-signaling pathway and an extension of lifespan in C57BL/6 mice [43]. Although currently there are no lifespan data on $\mathrm{Nrf}^{-/-}$mice, one could predict that their lifespan would be shortened compared to wild-type littermates.

Much of the data we find in naked mole-rats is consistent with data regarding Nrf2 in other models of healthy aging and extended longevity. We observe that naked mole-rat fibroblasts have 3-fold higher levels of Nrf2 in non-stressed animals compared to fibroblasts from mice, in addition to similarly elevated levels in Nrf2-regulated enzymes (fig. $4 \mathrm{a}-\mathrm{d}$ ). Nrf2 has been implicated in proteome maintenance, specifically by regulating subunits of the $26 \mathrm{~S}$ proteasome and thereby facilitating ubiquitinated protein degradation as well as through its regulation of the sequestosome ( $\mathrm{p} 62$ ), a protein involved in mediating organelle and protein degradation by autophagy. Both these processes are upregulated in the naked mole-rat and may contribute to highly efficient quality control of the proteome. Not surprisingly, given the high clearance rates of proteins that do not pass quality control, naked molerats have low levels of ubiquitinated proteins in their tissue homogenates [35]. The impeccable proteome maintenance that we previously observed thus may be largely due to the increased Nrf2 levels found in the mole-rats.

The superior resistance to a wide array of toxins we observe in fibroblasts isolated from naked mole-rats, in 
Fig. 4. The Nrf2-signaling cytoprotective pathway is elevated in naked mole-rat fibroblasts compared to those from mice. Constitutive Nrf2 protein levels are increased in the nucleus of naked mole-rat fibroblasts (a, b). Cells from the naked mole-rat also show an increase in Nrf2ARE binding activity (c) and increased NQO1 enzyme (an enzyme largely regulated by Nrf2) activity (d), demonstrating that not only do naked mole-rats have high levels of the Nrf2 protein, but this protein is active within the cell, potentially offering this species increased protection against a diverse array of potential stressors.

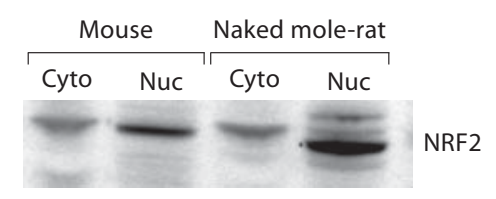

a
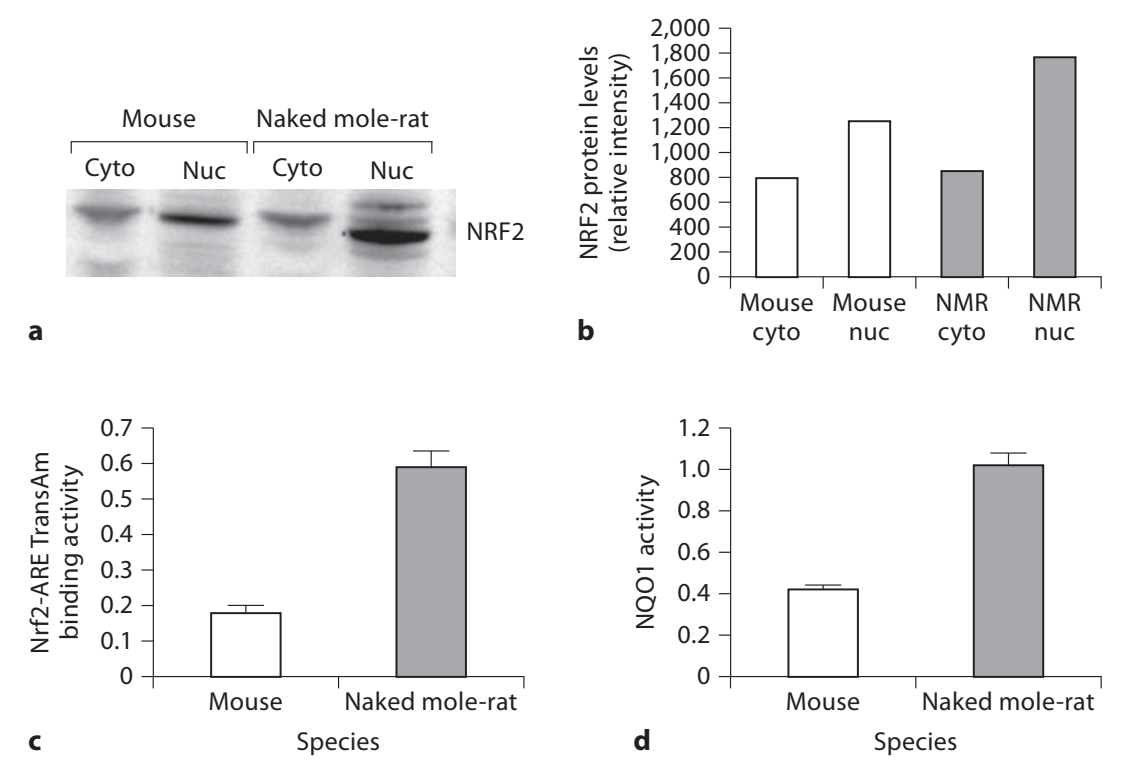

addition to preliminary results we have obtained in vivo, are most likely largely due to elevated Nrf2 signaling as well [44]. These trends are also magnified in vivo, as we have found naked mole-rats to be substantially more resistant to multiple hepatotoxins [unpubl. data], most likely due to their enhanced p53 and increased Nrf2 levels. Decline in vascular health often associated with age appears to be largely attenuated in the naked mole-rat in addition to signs of upregulated defense mechanisms against oxidative stress.

Nrf2 and p53 have also been shown to have a regulatory relationship during cell cycle response to stress [45]. p53 has been shown to inhibit Nrf2 transcription of genes in the ARE after a DNA-damaging event in the cell [46]. The two molecules appear to work together in the prevention of cancer. While the exact mechanism of this interaction is unknown, it is hypothesized that $\mathrm{p} 21$ plays a role as well [47]. Particularly interesting to us is that the naked mole-rat, an organism that is highly resistant to spontaneous tumors, has much higher levels of constitutively expressed p53 and Nrf2 compared to the shorter-lived and cancer-prone mouse, thus providing additional, albeit circumstantial evidence that both molecules may act in tandem to regulate the cell cycle, particularly after a toxic insult and thereby protect against cancer.

In summary, cytotoxic stress resistance has begun to emerge as a key indicator of positive healthspan and pro- longed longevity. Many of these cytoprotective pathways are highly conserved across many species. In particular, the Nrf2-signaling pathway has now been implicated in longevity and healthspan in evolutionary diverse phyla and may be central to the maintenance of good health well into old age.

\section{Acknowledgements}

This study was supported by grants to Rochelle Buffenstein (AFAR Breakthroughs in Gerontology, the Ellison Foundation, the Glenn Foundation and the NIA/NIH (AG022891-01)).

References 
5 Harper JM, Salmon AB, Leiser SF, Galecki AT, Miller RA: Skin-derived fibroblasts from long-lived species are resistant to some, but not all, lethal stresses and to the mitochondrial inhibitor rotenone. Aging Cell 2007;6:1-13.

-6 Buffenstein R: Negligible senescence in the longest living rodent, the naked mole-rat: insights from a successfully aging species. J Comp Physiol B 2008;178:439-445.

7 Ikeno Y, Hubbard GB, Lee S, Richardson A, Strong R, Diaz V, et al: Housing density does not influence the longevity effect of calorie restriction. J Gerontol A Biol Sci Med Sci 2005;60:1510-1517.

8 Liang ST, Mele J, Wu YH, Buffenstein R, Hornsby PJ: Resistance to experimental tumorigenesis in cells of a long-lived mammal, the naked mole-rat (Heterocephalus glaber). Aging Cell 2010;9:626-635.

-9 Seluanov A, Hine C, Azpurua J, Feigenson M, Bozzella M, Mao ZY, et al: Hypersensitivity to contact inhibition provides a clue to cancer resistance of naked mole-rat. Proc Natl Acad Sci USA 2009;106:19352-19357.

10 Buffenstein R, Kang J, Biney A: Glucose tolerance and insulin sensitivity in an extremely long-living rodent, the naked mole-rat. FASEB J 2007;21:A1423.

-11 Salmon AB, Akha AAS, Buffenstein R, Miller RA: Fibroblasts from naked mole-rats are resistant to multiple forms of cell injury, but sensitive to peroxide, ultraviolet light, and endoplasmic reticulum stress. J Gerontol A Biol Sci Med Sci 2008;63:232-241.

12 Brett RA: The population structure of naked mole-rat colonies; in Sherman PW, Jarvis J, Alexander R (eds): The Biology of the Naked Mole-Rat. Princeton, Princeton University Press, 1991, pp 97-136.

13 Buffenstein R: Ecophysiological responses to a subterranean habitat: a Bathyergid perspective. Mammalia 1996;60:591-605.

14 Buffenstein R, Yahav S: Is the naked mole-rat (Heterocephalus glaber) an endothermic yet poikilothermic mammal? J Therm Biol 1991; 16:227-232.

15 Buffenstein R, Yahav S: The effect of diet on microfaunal population and function in the cecum of a subterranean naked mole-rat (Heterocephalus glaber). Br J Nutr 1991;65: 249-258.

16 Rattan SIS: Theories of biological aging: genes, proteins, and free radicals. Free Radic Res 2006;40:1230-1238.

17 Sohal RS, Mockett RJ, Orr WC: Mechanisms of aging: an appraisal of the oxidative stress hypothesis. Free Radic Biol Med 2002;33: 575-586.

18 Perez VI, Bokov A, Van Remmen H, Mele J, Ran Q, Ikeno Y, Richardson A: Is the oxidative stress theory of aging dead? Biochim Biophys Acta 2009; 1790:1005-1014.

19 Salmon AB, Murakami S, Bartke A, Kopchick J, Yasumura K, Miller RA: Fibroblast cell lines from young adult mice of long-lived mutant strains are resistant to multiple forms of stress. Am J Physiol Endocrinol Metab 2005;289:E23-E29.
20 Allard JS, Heilbronn LK, Smith C, Hunt ND, Ingram DK, Ravussin E, et al: In vitro cellular adaptations of indicators of longevity in response to treatment with serum collected from humans on calorie restricted diets. PLoS One 2008;3:e3211.

21 Lithgow GJ, Benedetti MG, Foster AL, Vantipalli MC, White MP, Sampayo JN, et al: Compounds that confer thermal stress resistance and extended lifespan. Exp Gerontol 2008;43:882-891.

22 Sykiotis GP, Bohmann D: Keapl/Nrf2 signaling regulates oxidative stress tolerance and lifespan in Drosophila. Dev Cell 2008;14:7685.

23 Murakami S, Johnson TE: A genetic pathway conferring life extension and resistance to UV stress in Caenorhabditis elegans. Genetics 1996;143:1207-1218.

24 Ogburn CE, Austad SN, Holmes DJ, Kiklevich JV, Gollahon K, Rabinovitch PS, et al: Cultured renal epithelial cells from birds and mice: enhanced resistance of avian cells to oxidative stress and DNA damage. J Gerontol A Biol Sci Med Sci 1998;53:B287-B92.

25 De Cabo R, Furer-Galban S, Anson RM, Gilman C, Gorospe M, Lane MA: An in vitro model of caloric restriction. Exp Gerontol 2003;38:631-639.

26 Salmon AB, Leonard S, Masamsetti V, Pierce A, Podlutsky AJ, Podlutskaya N, et al: The long lifespan of two bat species is correlated with resistance to protein oxidation and enhanced protein homeostasis. FASEB J 2009; 23:2317-2326.

27 Cortopassi GA, Wang E: There is substantial agreement among interspecies estimates of DNA repair activity. Mech Ageing Dev 1996; 91:211-218.

28 Promislow DEL: On size and survival progress and pitfalls in the allometry of lifespan. J Gerontol 1993;48:B115-B123.

29 Babich H, Liebling EJ, Burger RF, Zuckerbraun HL, Schuck AG: Choice of DMEM, formulated with or without pyruvate, plays an important role in assessing the in vitro cytotoxicity of oxidants and prooxidant nutraceuticals. In Vitro Cell Dev Biol Anim 2009;45:226-233.

30 Wun WSA, Wun CCC, Grunert GM: Minimum essential medium- $\alpha$ enhances assisted reproductive technology results. I. Mouse embryo study. J Assist Reprod Gen 1994;11: 303-307.

31 Bishop CT, Mirza Z, Crapo JD, Freeman BA: Free-radical damage to cultured porcine aortic endothelial-cells and lung fibroblasts - modulation by culture conditions. In Vitro Cell Dev Biol Anim 1985;21:229-236.

32 Riley MR, Okeson CD, Riley-Saxton E: In vitro alveolar cytotoxicity of soluble components of airborne particulate matter: effects of serum on toxicity of transition metals. Toxicol In Vitro 2004;18:673-680.

33 Murakami S, Salmon A, Miller RA: Multiplex stress resistance in cells from longlived dwarf mice. FASEB J 2003;17:15651566.
Gage FH: Mammalian neural stem cells. Science 2000;287:1433-1438.

- 35 Perez VI, Buffenstein R, Masamsetti V, Leonard S, Salmon AB, Mele J, et al: Protein stability and resistance to oxidative stress are determinants of longevity in the longest-living rodent, the naked mole-rat. Proc Natl Acad Sci USA 2009;106:3059-3064.

36 McMahon M, Itoh K, Yamamoto M, Chanas SA, Henderson CJ, McLellan LI, et al: The cap ' $n$ ' collar basic leucine zipper transcription factor Nrf2 (NF-E2 p45-related factor 2) controls both constitutive and inducible expression of intestinal detoxification and glutathione biosynthetic enzymes. Cancer Res 2001;61:3299-3307.

-37 Kensler TW, Wakabayash N, Biswal S: Cell survival responses to environmental stresses via the Keap1-Nrf2-ARE pathway. Annu Rev Pharmacol 2007;47:89-116.

38 Hayes JD, McMahon M: NRF2 and KEAP1 mutations: permanent activation of an adaptive response in cancer. Trends Biochem Sci 2009;34:176-188.

- 39 Kraft AD, Johnson DA, Johnson JA: Nuclear factor E2-related factor 2-dependent antioxidant response element activation by tert-butylhydroquinone and sulforaphane occurring preferentially in astrocytes conditions neurons against oxidative insult. J Neurosci 2004;24:1101-1112.

40 Jasper H: SKNy worms and long life. Cell 2008;132:915-916.

-41 Brown-Borg HM, Rakoczy SG: Glutathione metabolism in long-living Ames dwarf mice. Exp Gerontol 2005;40:115-120.

42 Suh JH, Shenvi SV, Dixon BM, Liu HL, Jaiswal AK, Liu RM, et al: Decline in transcriptional activity of Nrf2 causes age-related loss of glutathione synthesis, which is reversible with lipoic acid. Proc Natl Acad Sci USA 2004;101:3381-3386.

43 Singh SP, Niemczyk M, Saini D, Sadovov V, Zimniak L, Zimniak P: Disruption of the mGsta4 gene increases life span of C57BL mice. J Gerontol A Biol Sci Med Sci 2010;65: 14-23.

44 Lewis KN, Mele J, Hayes JD, Buffenstein R: Nrf2, a guardian of healthspan and gatekeeper of species longevity. Integr Comp Biol 2010;50:829-843.

45 Piccirillo S, Filomeni G, Brune B, Rotilio G, Ciriolo MR: Redox mechanisms involved in the selective activation of Nrf2-mediated resistance versus p53-dependent apoptosis in adenocarcinoma cells. J Biol Chem 2009;284: 27721-27733.

46 Faraonio R, Vergara P, Di Marzo D, Pierantoni MG, Napolitano M, Russo T, et al: p53 suppresses the Nrf2-dependent transcription of antioxidant response genes. J Biol Chem 2006;281:39776-39784.

47 Toledano MB: The guardian recruits cops: the p53-p21 axis delegates prosurvival duties to the Keap1-Nrf2 stress pathway. Mol Cell 2009;34:637-639. 